\title{
On the kernel center of a convex body
}

\author{
Maria Moszyńska • E. Saorín Gómez
}

Received: 2 September 2013 / Accepted: 17 October 2013 / Published online: 6 November 2013 (C) The Author(s) 2013. This article is published with open access at Springerlink.com

\begin{abstract}
It is well known that the set of centers of minimal balls containing a convex body is a singleton, but the set of incenters of that body, i.e., its kernel, need not be a singleton. On the other hand, the kernel cannot have the same dimension as the body itself. By iterating the construction of the kernel we define a new selector, the kernel center, which selects a point from the kernel of a given convex body. Evidently, this selector is constant when restricted to the family of parallel bodies of a fixed convex body. We prove that it is directly additive but not additive, and we study further properties of this selector.
\end{abstract}

Keywords Convex body $\cdot$ Selector $\cdot$ Kernel $\cdot$ Inradius $\cdot$ Minkowski (direct) additivity Mathematics Subject Classification (1991) Primary 52A20; Secondary 52A99

\section{Introduction}

A selector for a family $\mathcal{X}$ of subsets of a metric space is a function on $\mathcal{X}$ which selects a point from every member of this family. For $n \geq 1$, we deal with the family $\mathcal{K}_{0}^{n}$ of convex bodies in $\mathrm{R}^{\mathrm{n}}$, that is, of compact convex subsets of $\mathrm{R}^{\mathrm{n}}$ with nonempty interiors.

Second author is supported by MEC and FEDER project MTM2011-25377 and by "Programa de Ayudas a Grupos de Excelencia de la Región de Murcia”, Fundación Séneca, 04540/GERM/06.

M. Moszyńska ( $\varangle)$

Institute of Mathematics, University of Warsaw, Warsaw, Poland

e-mail: mariamos@mimuw.edu.pl

\section{E. S. Gómez}

Institut für Algebra und Geometrie, Otto-von-Guericke Universität Magdeburg, Magdeburg, Germany e-mail: eugenia.saorin@ovgu.de 
Selectors for $\mathcal{K}_{0}^{n}$ have been studied by many authors (see Bárány 1988; Herburt 2002; Herburt and Moszyńska 2009; Moszyńska 1999, 2000, 2004, 2006; Moszyńska and Przesławski 1997; Moszyńska and Żukowski 1995; Schneider 1993, 1971).

The present paper concerns kernels of convex bodies in $\mathrm{R}^{\mathrm{n}}$. Using this notion, we define a new selector, the kernel center map, whose image belongs to the kernel of the convex body. This selector is constant when restricted to the family of inner parallel bodies of a given convex body (see next section for definitions). For a convex body $A$ this new selector is a relative of the Chebyshev center, $\check{c}$ (see Moszyńska 2006), which is the center of the unique ball in $\mathrm{R}^{\mathrm{n}}$ with minimal radius, containing $A$. Since, generally, for a convex body $A \subset \mathrm{R}^{\mathrm{n}}$ a ball with maximal radius contained in $A$ is not unique, there is no analogue to the Chebyshev center with balls containing $A$ replaced by balls contained in $A$. The kernel center map selects the incenter of one of the largest balls contained in a convex body. Of course, it coincides with its incenter if the convex body has a unique largest ball contained in it.

We warn the reader that the notion of kernel is commonly used for star bodies in a quite different meaning (see, for instance, Hansen and Martini 2011; Moszyńska 2004, 2006). For convex bodies these two notions of kernel differ essentially.

In Sect. 3 we study the Minkowski additivity and direct additivity of kernels of convex bodies. In Sect. 4 we focus on the kernel center map and prove that it is neither continuous with respect to the Hausdorff metric nor Minkowski additive. In turn, we prove that the kernel center map is directly additive (Theorem 4.1).

In the last section we compare the kernel center with some well known selectors: the centroid, the Steiner point, the center of the minimal ring, the Chebyshev center, and the pseudocenter, proving that the kernel center coincides, in general, with none of them.

\section{Preliminaries}

We use the following terminology and notation. Let $A$ be a nonempty subset of $\mathrm{R}^{\mathrm{n}}$. As usual, aff $\mathrm{A}$, lin $\mathrm{A}$, Int $\mathrm{A}$, relint $\mathrm{A}, \mathrm{cl} \mathrm{A}$, conv $\mathrm{A}$ are, respectively, the affine hull of $A$, the linear hull of $A$, the interior of $A$, the relative interior of $A$, the closure of $A$, and the convex hull of $A$. The (closed) unit ball of $\mathrm{R}^{\mathrm{n}}$ is denoted by $B^{n}$, and the Lebesgue measure by $\lambda_{n}$.

Let $\mathcal{K}^{n}$ be the family of nonempty compact convex subsets of $\mathrm{R}^{\mathrm{n}}$ and $\mathcal{K}_{0}^{n}:=\{A \in$ $\left.\mathcal{K}^{n} \mid \operatorname{Int} A \neq \emptyset\right\}$. Let $E$ be an affine subspace of $\mathrm{R}^{\mathrm{n}}$. The map $\pi_{E}: \mathrm{R}^{\mathrm{n}} \rightarrow E$ is the orthogonal projection of $\mathrm{R}^{\mathrm{n}}$ onto $E$ and $\mathcal{K}_{0}(E)=\left\{A \in \mathcal{K}^{n} \mid \operatorname{relint}_{E} A \neq \emptyset\right\}$.

For any nonempty $A_{1}, A_{2} \subset \mathrm{R}^{\mathrm{n}}$, the Minkowski sum, $A_{1}+A_{2}$, and the Minkowski difference, $A_{1} \dot{-} A_{2}$, are defined by

$$
\begin{aligned}
A_{1}+A_{2} & :=\left\{a_{1}+a_{2} \mid a_{j} \in A_{j} \text { for } j=1,2\right\} \text { and } \\
A_{1} \dot{-} A_{2} & :=\left\{x \in \mathrm{R}^{\mathrm{n}} \mid x+A_{2} \subset A_{1}\right\} .
\end{aligned}
$$

It follows from the definition that if $A_{1}, A_{2} \in \mathcal{K}_{0}^{n}$, then $A_{1}+A_{2} \in \mathcal{K}_{0}^{n}$, and if, moreover, $A_{2} \subset A_{1}$, then $A_{1} \dot{-} A_{2} \in \mathcal{K}^{n}$. 
For a convex body $A \in \mathcal{K}_{0}^{n}$, the inradius $\mathrm{r}(A)$ is defined by

$$
\mathrm{r}(A):=\sup \left\{r \mid \exists x \in \mathrm{R}^{\mathrm{n}}: x+r B^{n} \subset A\right\} .
$$

The kernel of $A, \operatorname{ker}(A)=A \dot{-} \mathrm{r}(A) B^{n}$, is the set of incenters of $A$. The dimension of $\operatorname{ker}(A)$ is strictly less than $n$ (see Bonnesen and Fenchel 1987, p. 59). The inner parallel body of $A$ at distance $0<\lambda<\mathrm{r}$ is the set $A \dot{-} \lambda B^{n}$.

Let $E_{1}, E_{2} \subset \mathrm{R}^{\mathrm{n}}$ be two orthogonal affine subspaces of $\mathrm{R}^{\mathrm{n}}$ with $\mathrm{R}^{\mathrm{n}}=E_{1}+E_{2}$. Then $\mathrm{R}^{\mathrm{n}}$ is the direct sum of $E_{1}$ and $E_{2}$, in symbols $E_{1} \oplus E_{2}$, and for any $A_{j} \in \mathcal{K}_{0}\left(E_{j}\right)$, $j=1,2$, the direct sum of $A_{1}, A_{2}$ is $A_{1} \oplus A_{2}:=A_{1}+A_{2} \in \mathcal{K}_{0}(E)$.

For $A_{1}, A_{2} \in \mathcal{K}^{n}$, the distance from $A_{1}$ to $A_{2}$ is given by the Hausdorff metric $\rho_{H}$.

Let $k \geq 2$. For affinely independent points $a_{1}, \ldots, a_{k} \in \mathrm{R}^{\mathrm{n}}$, let $\Delta\left(a_{1}, \ldots, a_{k}\right)$ be the simplex with vertices $a_{1}, \ldots, a_{k}$. Finally, $\left\{e_{1}, \ldots, e_{n}\right\}$ is the canonical basis in $\mathrm{R}^{\mathrm{n}}$.

\section{The kernel of a convex body}

The following result is evident.

Proposition 3.1 The function $\mathrm{ker}: \mathcal{K}_{0}^{n} \rightarrow \mathcal{K}^{n}$ is equivariant under isometries of $\mathrm{R}^{\mathrm{n}}$.

Remark 3.1 It is easy to see that the function ker is not equivariant under affine maps. It suffices to consider a cube and an orthogonal box with two edges of different lengths.

It is natural to ask whether the map ker is Minkowski additive. The following example shows that the answer is negative.

Example 3.1 Consider the following orthogonal boxes $A_{1}, A_{2} \in \mathcal{K}_{0}^{n}, A_{1}=$ $\Delta\left(-e_{1}, e_{1}\right)+\sum_{i=2}^{n} \Delta\left(-2 e_{i}, 2 e_{i}\right)$ and $A_{2}=\Delta\left(-2 e_{1}, 2 e_{1}\right)+\sum_{i=2}^{n} \Delta\left(-e_{i}, e_{i}\right)$. It is easy to check that $\operatorname{ker}\left(A_{1}\right)=\sum_{i=2}^{n} \Delta\left(-e_{i}, e_{i}\right)$ and $\operatorname{ker}\left(A_{2}\right)=\Delta\left(-e_{1}, e_{1}\right)$ while $\operatorname{ker}\left(A_{1}+A_{2}\right)=\{0\}$.

If the convex bodies lie in orthogonal affine flats, i.e., if we are dealing with direct sums, we can say more. For $A \in \mathcal{K}^{n}$ and $E=\operatorname{aff} A, \mathrm{r}_{E}$ and $\operatorname{ker}_{E}(A)$ denote, respectively, the inradius and the kernel of $A$ in $\mathcal{K}_{0}(E)$.

Theorem 3.1 Let $E_{1}, E_{2}$ be orthogonal flats with $\mathrm{R}^{\mathrm{n}}=E_{1} \oplus E_{2}$. Let $A_{j} \in \mathcal{K}_{0}\left(E_{j}\right)$ for $j=1,2$. Then

i) $\mathrm{r}\left(A_{1} \oplus A_{2}\right)=\min _{j=1,2} \mathrm{r}_{E_{j}}\left(A_{j}\right)$,

ii) $\operatorname{ker}_{E_{1}}\left(A_{1}\right)+\operatorname{ker}_{E_{2}}\left(A_{2}\right) \subset \operatorname{ker}_{E}\left(A_{1} \oplus A_{2}\right)$,

iii) $\operatorname{ker}_{E_{1}}\left(A_{1}\right)+\operatorname{ker}_{E_{2}}\left(A_{2}\right)=\operatorname{ker}_{E}\left(A_{1} \oplus A_{2}\right)$ if and only if $\mathrm{r}_{E_{1}}\left(A_{1}\right)=\mathrm{r}_{E_{2}}\left(A_{2}\right)$.

Proof For $j=1,2$, let $B_{j}=\pi_{E_{j}}\left(B^{n}\right)$. It is easy to see that $B^{n} \subset B_{1} \oplus B_{2}$.

Let $\mathrm{r}_{j}:=\mathrm{r}_{E_{j}}\left(A_{j}\right)$ for $j=1,2$ and $\mathrm{r}=\mathrm{r}\left(A_{1} \oplus A_{2}\right)$. We may assume that $\mathrm{r}_{1} \leq \mathrm{r}_{2}$.

i) Let $x \in \operatorname{ker}\left(A_{1} \oplus A_{2}\right)$; then $x+\mathrm{r} B^{n} \subset A_{1} \oplus A_{2}$. Projecting onto $E_{j}, j \in\{1,2\}$, we obtain

$$
\pi_{E_{j}}(x)+\mathrm{r} B_{j} \subset \pi_{E_{j}}\left(A_{1} \oplus A_{2}\right)=A_{j}
$$


and thus $\mathrm{r} \leq \mathrm{r}_{j}$ for $j \in\{1,2\}$. On the other hand, since $\mathrm{r}_{1} \leq \mathrm{r}_{2}$, it follows that $x_{j}+\mathrm{r}_{1} B_{j} \subset A_{j}$ for $x_{j} \in \operatorname{ker}_{E_{j}}\left(A_{j}\right), j=1,2$.

Hence,

$$
\left(x_{1}+x_{2}\right)+\mathrm{r}_{1} B^{n} \subset x_{1}+x_{2}+\mathrm{r}_{1}\left(B_{1} \oplus B_{2}\right) \subset A_{1} \oplus A_{2},
$$

which proves that $r_{1} \leq r$.

ii) From (3.1) it follows that if $x_{j} \in \operatorname{ker}_{E_{j}}\left(A_{j}\right), j=1,2$, then $x_{1}+x_{2} \in \operatorname{ker}\left(A_{1} \oplus\right.$ $\left.A_{2}\right)$.

iii) Assume first that $\mathrm{r}:=\mathrm{r}_{1}=\mathrm{r}_{2}$. In view of ii), it suffices to prove that $\operatorname{ker}\left(A_{1} \oplus\right.$ $\left.A_{2}\right) \subset \operatorname{ker}_{E_{1}}\left(A_{1}\right)+\operatorname{ker}_{E_{2}}\left(A_{2}\right)$.

Since $\operatorname{ker}_{E}\left(A_{1} \oplus A_{2}\right)+\mathrm{r} B^{n} \subset A_{1} \oplus A_{2}$, projecting onto $E_{j}$ for $j \in\{1,2\}$, we obtain

$$
\pi_{E_{j}}\left(\operatorname{ker}_{E}\left(A_{1} \oplus A_{2}\right)\right)+\mathrm{r}_{0} B_{j} \subset A_{j} .
$$

Hence, $\pi_{E_{j}}\left(\operatorname{ker}_{E}\left(A_{1} \oplus A_{2}\right)\right) \subset \operatorname{ker}_{E_{j}}\left(A_{j}\right)$ and

$$
\begin{array}{r}
\pi_{E_{1}}\left(\operatorname{ker}_{E}\left(A_{1} \oplus A_{2}\right)\right)+\pi_{E_{2}}\left(\operatorname{ker}_{E}\left(A_{1} \oplus A_{2}\right)\right) \\
=\operatorname{ker}\left(A_{1} \oplus A_{2}\right) \subset \operatorname{ker}_{E_{1}}\left(A_{1}\right)+\operatorname{ker}_{E_{2}}\left(A_{2}\right) .
\end{array}
$$

To prove the converse implication, assume that $\operatorname{ker}_{E_{1}}\left(A_{1}\right) \oplus \operatorname{ker}_{E_{2}}\left(A_{2}\right)=$ $\operatorname{ker}\left(A_{1} \oplus A_{2}\right)$. From $\left.i\right)$ we know that $\mathrm{r}\left(A_{1} \oplus A_{2}\right)=r_{1}$. Projecting onto $E_{j}$, we obtain $\pi_{E_{j}}\left(\operatorname{ker}\left(A_{1} \oplus A_{2}\right)\right)=\operatorname{ker}_{E_{j}} A_{j}$ for $j=1$, 2. If $\mathrm{r}_{1} \leq \mathrm{r}_{2}$, then

$$
\begin{aligned}
& \operatorname{ker}_{E_{1}}\left(A_{1}\right)+\operatorname{ker}_{E_{2}}\left(A_{2}\right)+\mathrm{r}_{1} B^{n}+\left(\mathrm{r}_{2}-\mathrm{r}_{1}\right) B^{n} \\
& \quad \subset \operatorname{ker}_{E_{1}}\left(A_{1}\right)+\mathrm{r}_{1}\left(B_{1}+B_{2}\right)+\left(\mathrm{r}_{2}-\mathrm{r}_{1}\right) B_{2} \subset A_{1} \oplus A_{2} .
\end{aligned}
$$

Thus we get

$$
\begin{aligned}
& \operatorname{ker}\left(A_{1} \oplus A_{2}\right)+\mathrm{r}_{1} B^{n}+\left(\mathrm{r}_{2}-\mathrm{r}_{1}\right) B_{2} \\
& \quad=\operatorname{ker}_{E_{1}}\left(A_{1}\right)+\operatorname{ker}_{E_{2}}\left(A_{2}\right)+\mathrm{r}_{1} B^{n}+\left(\mathrm{r}_{2}-\mathrm{r}_{1}\right) B_{2} \subset A_{1} \oplus A_{2},
\end{aligned}
$$

which implies $r_{1}=r_{2}$. This completes the proof.

Example 3.1 shows that, under the conditions of the previous theorem, if $\mathrm{r}_{E_{1}}\left(A_{1}\right) \neq$ $\mathrm{r}_{E_{2}}\left(A_{2}\right)$, then not only the inclusion $\operatorname{ker}_{E_{1}}\left(A_{1}\right)+\operatorname{ker}_{E_{2}}\left(A_{2}\right) \subset \operatorname{ker}_{E}\left(A_{1} \oplus A_{2}\right)$ must be strict, but also the inequality

$$
\operatorname{dim} \operatorname{ker}_{E_{1}}\left(A_{1}\right)+\operatorname{dim} \operatorname{ker}_{E_{2}}\left(A_{2}\right)<\operatorname{dim} \operatorname{ker}\left(A_{1}+A_{2}\right)
$$

may be strict.

We finish the section with the following remark which is a direct consequence of the definition of inner parallel bodies of $A \in \mathcal{K}_{0}^{n}$.

Remark 3.2 Let $\left\{A_{\lambda} \mid-\mathrm{r}(A) \leq \lambda \leq 0\right\}$ be the family of inner parallel bodies of $A \in \mathcal{K}^{n}$. Then $\operatorname{ker}\left(A_{\lambda}\right)=\operatorname{ker}(A)$ for all $\lambda \in[-\mathrm{r}, 0]$. 


\section{The kernel center of a convex body}

To any convex body $A \in \mathcal{K}_{0}^{n}$ we assign two finite sequences, $\left(\operatorname{ker}^{(i)}(A)\right)_{i \geq 0}$ and $\left(E_{i}(A)\right)_{i \geq 0}$, defined as follows:

$$
\operatorname{ker}^{(0)}(A):=\operatorname{ker}(A) \text { and } E_{0}(A):=\operatorname{aff} \operatorname{ker}(A)
$$

If $i \geq 1$ and $\operatorname{dim} \operatorname{ker}^{(i-1)}(A)>0$, then

$$
\operatorname{ker}^{(i)}(A):=\operatorname{ker}_{E_{i-1}(A)}\left(\operatorname{ker}^{(i-1)}(A)\right) \text { and } E_{i}(A):=\operatorname{aff}_{\operatorname{ker}^{(i)}}(A) .
$$

Let $m(A):=\min \left\{i \geq 0 \mid \operatorname{dim} \operatorname{ker}^{(i)}(A)=0\right\}$. Evidently, the sequences $\left(E_{i}(A)\right)_{i \geq 0}$ and $\left(\operatorname{ker}_{E_{i}}^{(i)}(A)\right)_{i \geq 0}$ are descending.

Moreover, if $\operatorname{dim} \operatorname{ker}^{(i)}(A)>0$, then $\operatorname{dim} \operatorname{ker}^{(i+1)}(A)<\operatorname{dim} \operatorname{ker}^{(i)}(A)$ (cf. Bonnesen and Fenchel 1987, p. 59).

Then, $\kappa(A)$ is defined as the unique point of $\operatorname{ker}^{m(A)}(A)$, or equivalently:

$$
\{\kappa(A)\}=\bigcap_{i=0}^{m(A)} \operatorname{ker}^{(i)}(A) .
$$

Of course $m(A) \in\{0, \ldots, n\}$, and it provides the number of steps needed to reach $\kappa(A)$ when passing from $\operatorname{ker}(A)$ to the subsequent kernels. It is clear that $\kappa(A) \in A$, hence, $\kappa: \mathcal{K}_{0}^{n} \rightarrow \mathrm{R}^{\mathrm{n}}$ is a selector for $\mathcal{K}_{0}^{n}$.

Remark 4.1 Let us notice that for any affine flat $E$ in $\mathrm{R}^{\mathrm{n}}$, with $\operatorname{dim} E=k \leq n-1$, the functions $\mathrm{r}: \mathcal{K}_{0}(E) \rightarrow \mathrm{R}^{\mathrm{n}}$, ker $: \mathcal{K}_{0}(E) \rightarrow \mathcal{K}^{n}$ and $\kappa: \mathcal{K}_{0}(E) \rightarrow \mathrm{R}^{\mathrm{n}}$ are well defined by identifying $E$ and $\mathrm{R}^{k}$.

Since the selector $\kappa$ is defined by means of the "subsequent kernels" of $A$, it is natural to ask whether $\kappa$ and ker behave in a similar way. To answer this, we deal next with some properties of $\kappa$. The following statement follows from Lemma 3.1.

Proposition 4.1 For every isometry $f: \mathrm{R}^{\mathrm{n}} \rightarrow \mathrm{R}^{\mathrm{n}}$ and every $A \in \mathcal{K}_{0}^{n}$,

$$
f(\kappa(A))=\kappa(f(A)) .
$$

As it happens with the kernel, the selector $\kappa$ is not equivariant under affine maps. In fact, it is not affine equivariant when restricted to the family of simplices. For this purpose, we notice that the incenter of a simplex $T$ coincides with $\kappa(T)$. In Edmonds et al. (2005) (Theorem 2.1) it is proven that the incenter of a simplex coincides with its centroid if and only if all the facets of the simplex have the same area. The centroid of a convex body is equivariant under affine transformations (see Moszyńska 2006, Theorem 12.3.8). Thus, it is enough to consider any simplex all whose facets do not have the same area, which of course is an affine image of a regular simplex.

Next we prove that $\kappa$ is not continuous with respect to the Hausdorff metric in $\mathrm{R}^{\mathrm{n}}$ for any $n \geq 2$. 
Proposition 4.2 The selector $\kappa: \mathcal{K}_{0}^{n} \rightarrow \mathrm{R}^{\mathrm{n}}$ is not continuous with respect to $\rho_{H}$.

Proof For every natural $k$, let

$$
A_{k}:=\operatorname{conv}\left(B^{n} \cup\left(2 e_{1}+\frac{k}{1+k} B^{n}\right)\right)
$$

and $A:=\operatorname{conv}\left(B^{n} \cup\left(2 e_{1}+B^{n}\right)\right)$. Evidently $A=\lim _{\rho_{H}} A_{k}$ while $e_{1}=\kappa(A) \neq$ $\kappa\left(A_{k}\right)=0$ for any $k$.

Let us prove the following statement concerning the kernel center map (cf. Example 3.1 ).

Proposition 4.3 The selector $\kappa: \mathcal{K}_{0}^{n} \rightarrow \mathrm{R}^{\mathrm{n}}$ is not Minkowski additive for any $n \geq 2$.

Proof Let $A_{1}$ be the orthogonal box $A_{1}=\left(\sum_{i=1}^{n-1} \Delta\left(-\rho e_{i}, \rho e_{i}\right)\right) \oplus \Delta\left(-e_{n}, e_{n}\right)$, for $2<\rho \in \mathbb{R}$ and $A_{2}:=\operatorname{conv}\left(B^{n} \cup\left\{2 \rho e_{n}\right\}\right)$. It is easy to check that $\operatorname{ker}\left(A_{1}\right)=$ $\sum_{i=1}^{n-1} \Delta\left(-\rho e_{i}, \rho e_{i}\right) \subset \operatorname{lin}\left\{e_{1}, \ldots, e_{n-1}\right\}$, whence $\kappa\left(A_{1}\right)=0$. On the other hand, $A_{2}$ has only one largest ball centered at the origin. Thus, $\kappa\left(A_{2}\right)=0$.

It suffices to prove that $0 \notin \operatorname{ker}\left(A_{1}+A_{2}\right)$. Let us consider the Minkowski sum $A_{1}+A_{2}=\left(\sum_{i=1}^{n-1} \Delta\left(-\rho e_{i}, \rho e_{i}\right)\right)+\Delta\left(-e_{n}, e_{n}\right)+\operatorname{conv}\left(B^{n} \cup\left\{2 \rho e_{n}\right\}\right)$. Since $\Delta\left(-e_{n}, 2 \rho e_{n}\right) \subset A_{2}$, it follows that

$$
\begin{aligned}
B: & =\left(\sum_{i=1}^{n-1} \Delta\left(-\rho e_{i}, \rho e_{i}\right)\right)+\Delta\left(-e_{n}, e_{n}\right)+\Delta\left(-e_{n}, 2 \rho e_{n}\right) \\
& =\left(\sum_{i=1}^{n-1} \Delta\left(-\rho e_{i}, \rho e_{i}\right)\right)+\Delta\left(-2 e_{n},(2 \rho+1) e_{n}\right) \subset A_{1}+A_{2} .
\end{aligned}
$$

On the other hand, $\mathrm{r}(B) \geq \rho$. Hence, there exists a ball of radius at least $\rho$ in $A_{1}+A_{2}$ while the largest ball centered at the origin and contained in $A_{1}+A_{2}$ has radius 2. Thus $0 \notin \operatorname{ker}\left(A_{1}+A_{2}\right)$, whence $\kappa\left(A_{1}+A_{2}\right) \neq 0$.

The selector $\kappa$ exhibits a nice behavior when dealing with direct sum.

Theorem 4.1 Let $E_{1}, E_{2}$ be orthogonal affine flats with $\mathrm{R}^{\mathrm{n}}=E_{1} \oplus E_{2}$. Let $A_{1} \in$ $\mathcal{K}_{0}^{n}\left(E_{1}\right)$ and $A_{2} \in \mathcal{K}_{0}^{n}\left(E_{2}\right)$. Then

$$
\kappa\left(A_{1} \oplus A_{2}\right)=\kappa\left(A_{1}\right)+\kappa\left(A_{2}\right) .
$$

Proof Let $\mathrm{r}_{1}=\mathrm{r}_{E_{1}}\left(A_{1}\right) \leq \mathrm{r}_{E_{2}}\left(A_{2}\right)=\mathrm{r}_{2}$ and $\operatorname{dim} E_{1}=k$. By Theorem 3.1,

$$
\mathrm{r}\left(A_{1} \oplus A_{2}\right)=\min \left\{\mathrm{r}_{1}, \mathrm{r}_{2}\right\}=\mathrm{r}_{1}
$$

Let us prove that

$$
\operatorname{ker}\left(A_{1} \oplus A_{2}\right)=\operatorname{ker}_{E_{1}}\left(A_{1}\right)+\left(A_{2}\right)_{-\mathrm{r}_{1}} .
$$


Indeed

$$
\operatorname{ker}_{E_{1}}\left(A_{1}\right)+\left(A_{2}\right)_{-\mathrm{r}_{1}}+\mathrm{r}_{1} B^{n} \subset A_{1} \oplus A_{2}
$$

thus,

$$
\operatorname{ker}_{E_{1}}\left(A_{1}\right)+\left(A_{2}\right)_{-\mathrm{r}_{1}} \subset \operatorname{ker}\left(A_{1} \oplus A_{2}\right)
$$

On the other hand,

$$
\operatorname{ker}\left(A_{1} \oplus A_{2}\right)+\mathrm{r}_{1} B^{n} \subset A_{1} \oplus A_{2} .
$$

Projecting onto $E_{j}, j=1,2$, we obtain $\pi_{E_{1}}\left(\operatorname{ker}\left(A_{1} \oplus A_{2}\right)\right) \subset \operatorname{ker}_{E_{1}}\left(A_{1}\right)$ and $\pi_{E_{2}}\left(\operatorname{ker}\left(A_{2} \oplus A_{2}\right)\right) \subset\left(A_{2}\right)_{-r_{1}}$. Hence

$$
\operatorname{ker}\left(A_{1} \oplus A_{2}\right)=\operatorname{ker}_{E_{1}}\left(A_{1}\right)+\left(A_{2}\right)_{-\mathrm{r}_{1}},
$$

which proves (4.4).

It is clear that $\operatorname{dim} \operatorname{ker}_{E_{1}}\left(A_{1}\right)<k$. Since in each step the dimension of one of the two summands decreases, in order to get $\kappa\left(A_{1} \oplus A_{2}\right)$ we need to iterate this process a finite number of steps. After $i$ iterations we will have, for $l, m \in\{1,2\}$, $l \neq m$, and $j \in\{1, \ldots, i-1\}$, one of the following Minkowski sums: $\left(A_{l}\right)_{\mu}+$ $\operatorname{ker}_{E_{m}}^{(i-1)}\left(A_{m}\right)$ for some $-\mathrm{r}_{E_{l}}\left(A_{l}\right)<\mu<0$ or $\operatorname{ker}_{E_{l}}^{j}\left(A_{l}\right)+\operatorname{ker}_{E_{m}}^{i-1-j}\left(A_{m}\right)$. By Remark $3.2, \operatorname{ker}_{E_{l}}\left(\left(A_{l}\right)_{\mu}\right)=\operatorname{ker}\left(A_{l}\right)$. Thus, after at most $m_{E_{1}}\left(A_{1}\right)+m_{E_{2}}\left(A_{2}\right)+1$ steps we obtain $\kappa\left(A_{1} \oplus A_{2}\right)=\kappa\left(A_{1}\right)+\kappa\left(A_{2}\right)$.

\section{Final remarks}

We compare the kernel center map with some well known selectors.

Proposition 5.1 The kernel center map $\kappa$ is different from the Steiner point map $s$, the Chebyshev center $\check{c}$, the centroid $c_{0}$, the center of the minimal ring $c$, and the pseudocenter $p$.

Proof (1) Let $s$ be the Steiner point map, that is

$$
s(A):=\frac{1}{\lambda_{n}\left(B^{n}\right)} \int_{S^{n-1}} u h_{A}(u) d \sigma(u),
$$

where $h_{A}$ is the support function of the convex body $A$. Since the Steiner point map $s$ is continuous with respect to $\rho_{H}$ and Minkowski additive (see e.g. Schneider 1971), in view of Propositions 4.2 and $4.3, \kappa \neq s$.

(2) Let $\breve{c}(A)$ be the Chebyshev center of $A$, i.e., the center of the unique ball with minimal radius containing $A$. Let $A$ be the cone over the $(n-1)$-dimensional ball $B^{n} \cap\left(\text { lin } e_{n}\right)^{\perp}$, with vertex $e_{n}$. Then $\check{c}(A)=0 \in \operatorname{bd} A$, while $\kappa(A) \in \operatorname{int} A$. Thus $\kappa \neq \check{c}$. 
(3) Let $c_{0}(A)$ be the centroid of $A$. By Edmonds et al. (2005) (Theorem 3.2) the centroid and the incenter of a simplex coincide if and only if all the facets of the simplex have the same area. Hence, $\kappa \neq c_{0}$.

(4) Let $c(A)$ be center of the minimal ring containing $A$, that is, the minimizer of $R_{A}(x)-r_{A}(x)$, where $R_{A}(x)$ is the minimal radius of a ball with center $x$ containing $A$ and $r_{A}(x)$ is the maximal radius of a ball with center $x$ contained in $A$ (cf. Bonnesen and Fenchel 1987; Moszyńska 2006). Since the selector $c$ is continuous with respect to the Hausdorff metric (Moszyńska 2006, Theorem 12.5.8), from Proposition 4.2 it follows that $\kappa \neq c$.

(5) Let $p(A)$ be the pseudocenter of $A$, i.e., the symmetry center of the centrally symmetric convex body with maximal volume contained in $A$. The selector $p$ is equivariant under affine maps (see Moszyńska 2006, Th. 12.6.3), while the kernel center map is not. Thus $\kappa \neq p$.

Open Access This article is distributed under the terms of the Creative Commons Attribution License which permits any use, distribution, and reproduction in any medium, provided the original author(s) and the source are credited.

\section{References}

Bárány, I.: On the minimal ring containing the boundary of a convex body. Acta Sci. Math. 52, 93-100 (1988)

Bonnesen, T., Fenchel, W.: Theorie der konvexen Körper, Springer, Berlin, 1934, 1974. English translation: Theory of convex bodies (eds., Boron, L., Christenson, C., Smith, B.), BCS Associates, Moscow (1987)

Edmonds, A.L., Hajja, M., Martini, H.: Coincidences of simplex centers and related facial structures. Beiträge Algebra Geom. 46(2), 491-512 (2005)

Hansen, G., Martini, H.: Starshapedness vs. convexity. Results Math. 59, 185-197 (2011)

Herburt, I.: On the Lipschitz continuity of the center of the minimaln ring. Rend. Circ. Mat. Palermo, Serie II. 70, 385-393 (2002)

Herburt, I., Moszyńska, M.: Optimal isometries for a pair of compact convex subsets of $\mathrm{R}^{n}$. In: Convex and Fractal Geometry. Institute of Mathematics, Polish Academy of Sciences, vol. 84, pp. 111-120 Banach Center Publications (2009)

Moszyńska, M.: Selected topics in convex geometry. Birkhäuser, Boston (2006)

Moszyńska, M.: Remarks on the minimal rings of convex bodies. Studia Sci. Math. Hung. 35, 155-174 (1999)

Moszyńska, M.: Looking for selectors of star bodies. Geom. Ded. 83, 131-147 (2000)

Moszyńska, M.: On directly additive selectors for convex and star bodies. Glasnik Mat. 39(59), 145-154 (2004)

Moszyńska, M., Przesławski, K.: Santaló points associated with radial densities. Rend. Circ. Mat. Palermo. 50(2), 273-288 (1997)

Moszyńska, M., Żukowski, T.: Duality of convex bodies. Geom. Ded. 58, 161-173 (1995)

Schneider, R.: Convex bodies: the Brunn-Minkowski Theory. Cambridge University Press, Cambridge (1993)

Schneider, R.: On Steiner points of convex bodies. Israel J. Math. 9, 241-249 (1971) 